\title{
A spheroid-based 3-D culture model for pancreatic cancer drug testing, using the acid phosphatase assay
}

\author{
Z. Wen, Q. Liao, Y. Hu, L. You, L. Zhou and Y. Zhao \\ Department of General Surgery, Peking Union Medical College Hospital, \\ Chinese Academy of Medical Science and Peking Union Medical College, Tsinghua University, Beijing, China
}

\begin{abstract}
Current therapy for pancreatic cancer is multimodal, involving surgery and chemotherapy. However, development of pancreatic cancer therapies requires a thorough evaluation of drug efficacy in vitro before animal testing and subsequent clinical trials. Compared to two-dimensional culture of cell monolayer, three-dimensional (3-D) models more closely mimic native tissues, since the tumor microenvironment established in 3-D models often plays a significant role in cancer progression and cellular responses to the drugs. Accumulating evidence has highlighted the benefits of 3-D in vitro models of various cancers. In the present study, we have developed a spheroid-based, 3-D culture of pancreatic cancer cell lines MIAPaCa-2 and PANC-1 for pancreatic drug testing, using the acid phosphatase assay. Drug efficacy testing showed that spheroids had much higher drug resistance than monolayers. This model, which is characteristically reproducible and easy and offers rapid handling, is the preferred choice for filling the gap between monolayer cell cultures and in vivo models in the process of drug development and testing for pancreatic cancer.
\end{abstract}

Key words: Spheroid; Three-dimensional culture; Pancreatic cancer; Drug testing

\section{Introduction}

Pancreatic cancer has a poor prognosis. For about the last 10 years, gemcitabine has remained the first-line chemotherapeutic agent for advanced pancreatic cancer; however, the success of drug treatment is poor, and overall survival has not improved for decades. Drug resistance is thought to be a major reason for the limited benefit of most pancreatic cancer therapies. Increasing drug efficiency and decreasing drug resistance are current principal aims in pancreatic cancer research (1).

The most common drug model for in vitro study of cancer cells is the monolayer culture of cells. Although the two-dimensional (2-D) model has made significant contributions to cancer research, it has certain intrinsic limitations that have promoted the development of threedimensional (3-D) culture models. Compared to 2-D culture models, 3-D culture models can provide a microenvironment that more closely mimics the microenvironment observed in tumor tissues. This feature is crucial for drug testing, since environmental cues can have profound effects on properties, behaviors, and functions of cancer cells, which may in turn affect cellular responses to drugs (2-4). Thus, 3-D culture models offer a more sophisticated means of mimicking in vivo environments, including the control of concentration gradients of signaling molecules and therapeutic agents, composition and structure of extracellular matrix surrounding the cancer cells, and the morphology and arrangement of individual cells.

Multicellular spheroids are probably the most widely accepted model for 3-D culture (5). Several studies have highlighted the potential of multicellular tumor spheroids (MCTS) in cancer research and treatment (6-12). Multicellular spheroid cultures can reflect the tumor microenvironment, volume growth kinetics, and cytoarchitecture, similar to those of avascular tumor nodules, micrometastasis, or the intervascular region of large, solid tumors. Previous research has studied MCTS-based drug screening in various types of tumors $(13,14)$. MCTS in pancreatic cancer were first described by McLeod (15). However, MCTS remain poorly investigated in spheroid-based chemotherapy research.

Correspondence: Q. Liao and/or Y. Zhao, Department of General Surgery, Peking Union Medical College Hospital, Chinese Academy of Medical Science and Peking Union Medical College, Tsinghua University, 1 Shuai Fu Yuan Hu Tong, Beijing 100730, China. E-mail: Iqpumc@yahoo.com.cn and/or zhao8028@263.net 
In the present study, a spheroid-based 3-D culture model of MIAPaCa-2 and PANC-1 MCTS, using the acid phosphatase assay, was established to investigate the chemotherapy characteristics of pancreatic cancer cells. As an important supplement to monolayer-based assays, well-controlled MCTS may provide new insights and a better estimation of antitumor efficacy for pancreatic cancer drug testing.

\section{Material and Methods}

\section{Cell lines and spheroid culture}

The MIAPaCa-2 and PANC-1 human pancreatic cancer cells were the kind gift of Professor Helmut Friess at the Department of Surgery, Klinikum Rechts der Isar, Technische Universität München, Germany. Cells were thawed from frozen stock and subcultured for $<20$ passages. Dulbecco's modified Eagle's medium (DMEM) containing $1 \mathrm{~g} / \mathrm{L}$ glucose, $1 \%$ (w/v) sodium pyruvate, $1 \%(\mathrm{w} / \mathrm{v})$ L-glutamine, and $3.7 \%(\mathrm{w} / \mathrm{v})$ $\mathrm{NaHCO}_{3}$, supplemented with $100 \mathrm{U} / \mathrm{mL}$ penicillin, $100 \mu \mathrm{g} / \mathrm{mL}$ streptomycin, and $10 \%$ fetal calf serum (FCS), was used for culturing (HyClone, USA). All cultures were kept at $37^{\circ} \mathrm{C}$ in a humidified atmosphere with $5 \% \mathrm{CO}_{2}$. Cell transfer and preparation of single-cell suspensions were performed by mild enzymatic dissociation, using $0.05 \%$ trypsin and $0.02 \%$ EDTA in phosphatebuffered saline (HyClone). Spheroids were initiated in a liquid overlay by seeding $1.2 \times 10^{3} \mathrm{MIAPaCa}-2$ cells and $1.0 \times 10^{3}$ PANC-1 cells/well in $200 \mu \mathrm{L}$ medium using agarose-coated, 96-well culture plates $(50 \mu \mathrm{L} 1.5 \%$ agarose/well). After an initiation interval of 4 days, $50 \%$ of supernatant was replaced with fresh medium, repeated every $48 \mathrm{~h}$ thereafter except for a $72 \mathrm{~h}$ drug treatment setup described in the drug treatment section.

\section{Cell counting, flow cytometry, and microscopy}

For the detection of viable cells, 15 to 30 spheroids were collected and dissociated using a spheroid dispersion solution (SCIVAX, Inc., USA), and the cell number was counted with a NucleoCounter (Chemometec, Denmark). Cell aliquots were stained with the DNA intercalating dye propidium iodide (PI, $2 \mu \mathrm{g} / \mathrm{mL}$ per $10^{6}$ cells), and the cells were analyzed on an Accuri C6 Flow Cytometer using CFlow Plus software packages (Accuri Cytometers, Inc., USA).

Morphological analyses of spheroids and phase contrast imaging in 96-well microplates were carried out manually on a DM IL inverted phase-contrast microscope (Leica, Germany). The volumes of the spheroids were calculated as previously described $(16,17)$. Generated data tables contained the following morphometric information: maximum diameter $(d 1)$, the diameter at right angles to maximum diameter $(d 2)$, mean spheroid diameter $(D)$, and spheroid volume. Volumes were calculated with the AVD mass and volume calculator
7.2.1 (Avlan Design, Canada). Briefly, $d 1$ and $d 2$ were measured and recorded. $D$ was calculated using the formula $D=\sqrt{d 1 d 2}$. Spheroid volume $\left(\mathrm{mm}^{3}\right)$ was calculated using the formula $\mathrm{V}=\frac{4}{3} \pi\left(\frac{D}{2}\right)^{3}$.

\section{Acid phosphatase assay}

A modified acid phosphatase (APH) assay, which was based on quantification of cytosolic APH activity, was validated for determining viable cells in spheroids (18). Intracellular APH in viable cells hydrolyzed $p$-nitrophenyl phosphate to $p$-nitrophenol. Its absorbance at $405 \mathrm{~nm}$ was directly proportional to the cell number in the range of $10^{3}$ to $10^{5}$ cells/monolayer. MCTS cultures grown in liquid overlay were transferred with supernatant onto flatbottom, 96-well microplates and centrifuged for $10 \mathrm{~min}$ at $1500 \mathrm{~g}$ to spin down spheroids, clusters, and single cells. The pellet was washed by carefully replacing $160 \mu \mathrm{L}$ of supernatant with phosphate buffer solution (PBS). Centrifugation was repeated, and the supernatant was discarded to a final volume of $100 \mu \mathrm{L}$. Then, $100 \mu \mathrm{L}$ of assay buffer was added per well and incubated for $90 \mathrm{~min}$ at $37^{\circ} \mathrm{C}$. Following incubation, $10 \mu \mathrm{L} \mathrm{NaOH}$ was added to each well, and absorbance at $405 \mathrm{~nm}$ was measured within 10 min with a WellScan MK3 microplate reader (Labsystems Dragon, Finland).

\section{Drug treatment}

Treatment was performed with 5-fluorouracil (5-FU, Sigma-Aldrich, USA) and gemcitabine hydrochloride (Eli Lilly and Company, USA). Drugs (100 mM stock solutions in dimethyl sulfoxide) were applied in the following concentrations: $200,100,50,25,10,1,0.1$, and $0.01 \mu \mathrm{M}$, in culture medium. Treatment with $10 \%$ Triton-X-100 (Sigma-Aldrich) in culture medium for $1.5 \mathrm{~h}$ at $37^{\circ} \mathrm{C}$ led to a $100 \%$ loss of cell membrane integrity in structurally intact spheroids, and served as positive control. For treatment, MIAPaCa-2 and PANC-1 monolayer cultures were grown in 96-well plates by plating $5 \times 10^{2}$ exponentially growing cells per well. Spheroids were grown in liquid overlay, as described above. Both monolayer and spheroid cultures were treated for $96 \mathrm{~h}$ after inoculation by replacing $50 \%$ $(100 \mu \mathrm{L})$ of the culture supernatant with drug-supplemented fresh medium. The spheroid diameter at the onset of treatment was 370 to $410 \mu \mathrm{m}$. The treatment interval was $72 \mathrm{~h}$. Untreated control cells and spheroids were always cultured in parallel, using $100 \mu \mathrm{L}$ dimethyl sulfoxidecontaining medium for the drug dilution.

\section{Analysis of drug efficacy}

Drug effects were documented after $72 \mathrm{~h}$ of treatment via the APH assay and spheroid volume analysis. All experiments were carried out in triplicate. APH data were corrected for background absorption at $405 \mathrm{~nm}$. Drug effects were recorded relative to untreated controls, using the mean APH signal of untreated spheroids $(n=9)$ measured for each individual experiment. 


\section{Statistical analysis}

Dose-response curves and $\mathrm{IC}_{50}$ values were calculated for each individual experiment by sigmoidal doseresponse analysis, using the Hill fitting equation in the Prism 5 software (GraphPad Software, Inc., USA). The individual dose-response curves and $\mathrm{IC}_{50}$ values for each drug were averaged, and are reported as means \pm SD.

\section{Results}

Characteristics of MIAPaCa-2 and PANC-1 pancreatic cancer spheroids

Single MCTS of about $400 \mu \mathrm{m}$ in diameter were harvested after a 96-h initiation incubation of $1.2 \times 10^{3}$ MIAPaCa- 2 cells and $1.0 \times 10^{3}$ PANC- 1 cells per culture well. With the medium being refreshed every $48 \mathrm{~h}$, spheroid volume increased according to the Gomertz equation, which classically and mathematically describes tumor and also spheroid growth kinetics (19). Maximum spheroid diameter of about $1 \mathrm{~mm}$ was reached after about 9 days for MIAPaCa- 2 and 10 days for PANC- 1 spheroids. Double spheroid volume was obtained after $29 \mathrm{~h}$ for MIAPaCa-2 and $28 \mathrm{~h}$ for PANC-1 spheroids (Figure 1A).

We compared the dead cells, calculated as the proportion of PI-stained cells in spheroids, as determined by flow cytometric analysis following dissociation (Figure 1B). The proportion of dead cells in MIAPaCa-2 spheroids of 400 and $600 \mu \mathrm{m}$ in size was about 5 to $8.58 \%$ and 15.52 to $20.02 \%$, respectively. On the other hand, the proportion of dead cells in PANC-1 spheroids with sizes of 400 and $600 \mu \mathrm{m}$ was about 4.96 to $10 \%$ and 15.78 to $19.86 \%$, respectively.

The number of viable cells per spheroid, was determined by automated counting following spheroid dissociation. For MIAPaCa-2 and PANC-1, a positive linear correlation between spheroid size and viable cells per spheroid was observed (Figure 1C). Meanwhile, the proportion of PI-positive cells (membrane-defective cells) monitored by flow cytometry increased during spheroid growth, reflecting the initiation of more cell death in the spheroid center at sizes $>500 \mu \mathrm{m}$. On the other hand, spheroids of smaller size (e.g., $400 \mu \mathrm{m}$ ) contained only 5 to $10 \%$ membrane-defective cells.

\section{Applicability and linearity of the APH assay in pancreatic cancer cell spheroid cultures}

The linearity of the APH assay signal in MIAPaCa-2 and PANC-1 spheroids as a function of the viable cell count/spheroid is shown in Figure 2. The absorption signal was linear in MIAPaCa-2 spheroids, with cell number from $3.3 \times 10^{3}$ to $1.24 \times 10^{5}$ cells/spheroid, covering spheroid diameters of up to $1000 \mu \mathrm{m}$. In PANC-1 spheroids, the APH signal was linear up to a maximum cell number of $1.19 \times 10^{5}$ cells/spheroid and a diameter of about $1000 \mu \mathrm{m}$. These results showed applicability and well-fitting linearity of the APH assay in both MIAPaCa-2 and PANC-1 spheroid cultures

\section{Cytotoxicity in MCTS using the APH assay}

In order to investigate the applicability of the $\mathrm{APH}$ method for determining drug-induced cytotoxicity in spheroids, MIAPaCa-2 and PANC-1 MCTS at day 4 with a size of 360 to $410 \mu \mathrm{m}$, that consisted of about 0.78 to $1.5 \times 10^{4}$ and 0.70 to $1.5 \times 10^{4}$ viable cells and $<10 \%$ dead cells, were incubated with gemcitabine. Spheroids were analyzed after a 72-h treatment interval with 0.01 to $200 \mu \mathrm{M}$ gemcitabine for the following parameters: spheroid size and volume, APH-assay signal, and cell count per spheroid. The effect of gemcitabine relative to untreated controls was calculated, and the results of the APH assay and cell count analysis were elevated (Figure 3). The results showed quite similar curves across the gemcitabine concentration range that was applied here. The results further indicated that the APH assay would be a reliable and valuable model to investigate drug efficacy in spheroids.

\section{Drug efficacy in MCTS vs monolayer culture using the APH assay}

Drug efficacy in 3-D cultures was revealed to be different from that observed in classical 2-D cell cultures. The APH assay is a novel tool used to investigate and quantify differences of drug sensitivity in spheroid and monolayer cultures. In this study, two drugs (gemcitabine and 5-FU) were compared in relation to application of the APH assay in spheroids. The drug dilutions used did not alter the background absorbance. Dose-response curves were documented in MIAPaCa-2 as well as PANC-1 monolayer cultures and in spheroids ranging in size from 360 to $410 \mu \mathrm{m}$ at the initiation of treatment. Drug efficacy experiments were performed on a 96-well plate using 0.01 to $200 \mu \mathrm{M}$ gemcitabine and 5-FU.

Drug effects in MIAPaCa-2 and PANC-1 cells were elevated after 72-h treatment intervals in three independent experiments per drug with $n=8$ spheroids per condition in each experiment. After a 72-h drug treatment, the APH signal decreased in a dose-dependent manner relative to untreated controls. Treatment with $200 \mu \mathrm{M}$ gemcitabine of MIAPaCa- 2 cells resulted in $>64 \%$ loss of cell viability, whereas PANC-1 monolayer cells generally showed a $45 \%$ survival rate, even at high gemcitabine concentrations. When treated with $200 \mu \mathrm{M} 5-\mathrm{FU}$, MIAPaCa-2 and PANC-1 monolayer cells showed a $38.6 \%$ and $51.24 \%$ survival rate, respectively. The $I_{50}$ values in MIAPaCa-2 monolayer cultures were $47.6 \pm 25.2 \mu \mathrm{M}$ for gemcitabine and $61.9 \pm 21.2 \mu \mathrm{M}$ for 5 -FU. PANC-1 monolayer cultures showed $\mathrm{IC}_{50}$ values of $64.9 \pm 10.3 \mu \mathrm{M}$ for gemcitabine. At the highest drug concentration of $200 \mu \mathrm{M}$, a reduction of cell viability $>50 \%$ was not observed; thus the $I_{50}$ in PANC- 1 monolayer culture could not be estimated for 5-FU (Figure 4). 

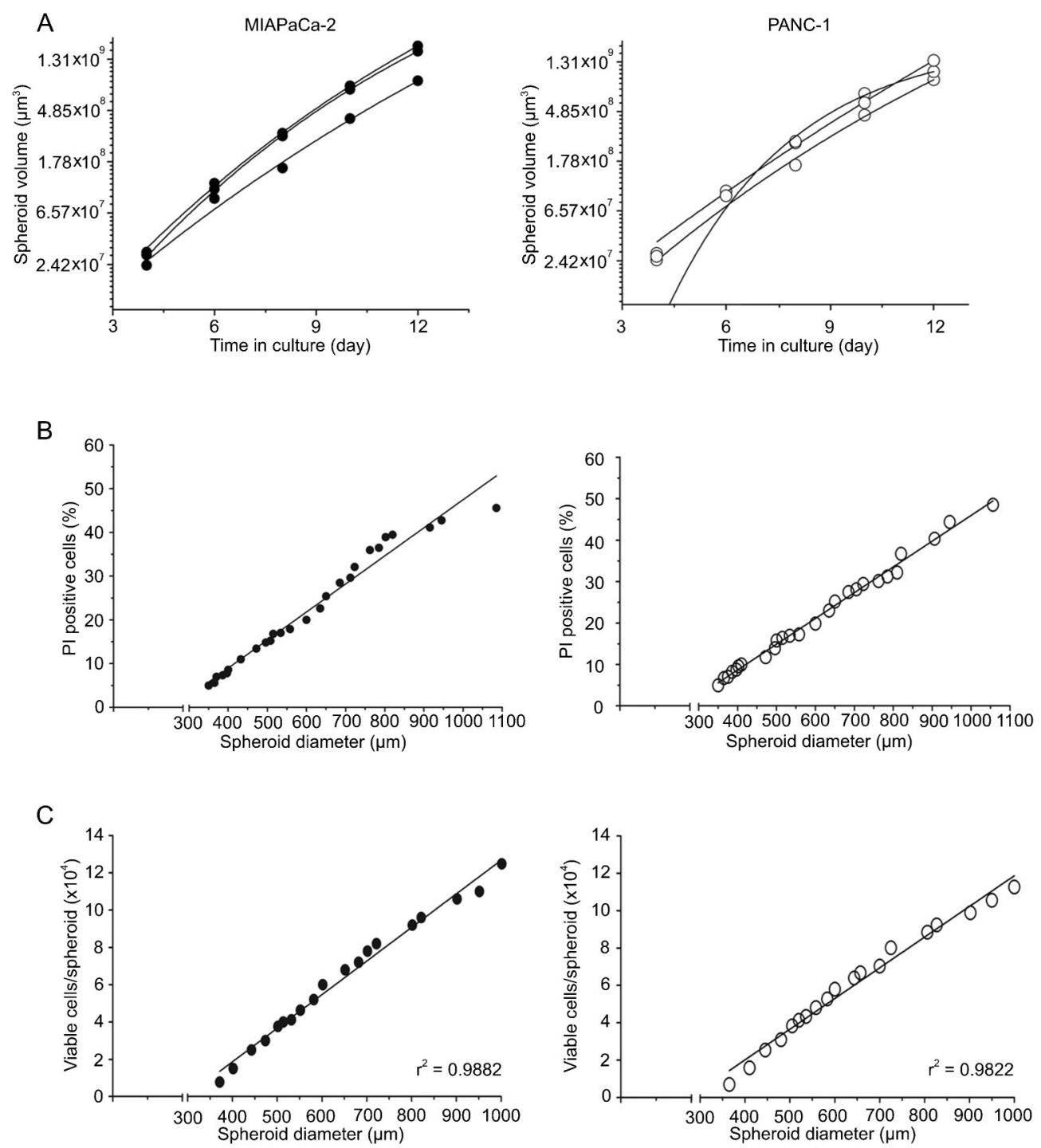

Figure 1. General characteristics of spheroid growth. A, Volume of MIAPaCa-2 and PANC-1 spheroids as a function of time in culture, with an initiation interval of 4 days and a seeding density of $1.2 \times 10^{3}$ MIAPaCa- 2 cells and $1.0 \times 10^{3}$ PANC- 1 cells per well. Data points are mean spheroid volumes for 8 to 16 spheroids. B. Proportion of propidium iodide (PI)-positive cells in MIAPaCa-2 and PANC-1 spheroids as a function of the average spheroid diameter determined by flow cytometry following dissociation of 15 to 30 spheroids. $C$, Viable cells in MIAPaCa-2 and PANC-1 spheroids as a function of the average spheroid diameter. Data are reported as average cell numbers determined from 3 aliquots of 15 to 30 spheroids.

MIAPaCa-2 and PANC-1 spheroid cultures demonstrated significantly reduced sensitivities to two different chemotherapeutic agents. Even at the highest drug concentration of $200 \mu \mathrm{M}, a \geqslant 50 \%$ reduction of cell viability was not observed in either MIAPaCa-2 or PANC-1 spheroids (Figure 4). Thus, the $\mathrm{IC}_{50}$ in MIAPaCa-2 and PANC-1 spheroid cultures could not be estimated for gemcitabine and 5-FU. These results showed that the efficacy of the drugs tested in this study was generally lower in MIAPaCa-2 and PANC-1 spheroids than in monolayer culture.
Microscopic imaging was performed prior to the APH assay in order to test the hypothesis that the spheroid volume after treatment was not necessarily indicative of cell viability and the signal intensity of the APH assay. Phase contrast images of MIAPaCa-2 spheroids treated with gemcitabine and 5-FU are shown in Figure 5. Spheroid volume reduction and disruption reflected a cell loss. Evident spheroid shedding and disruption occurred in spheroids of MIAPaCa-2 cells treated with $100 \mu \mathrm{M}$ gemcitabine, but viable cells could still be detected by the APH assay. Nevertheless, microscopic images might still 

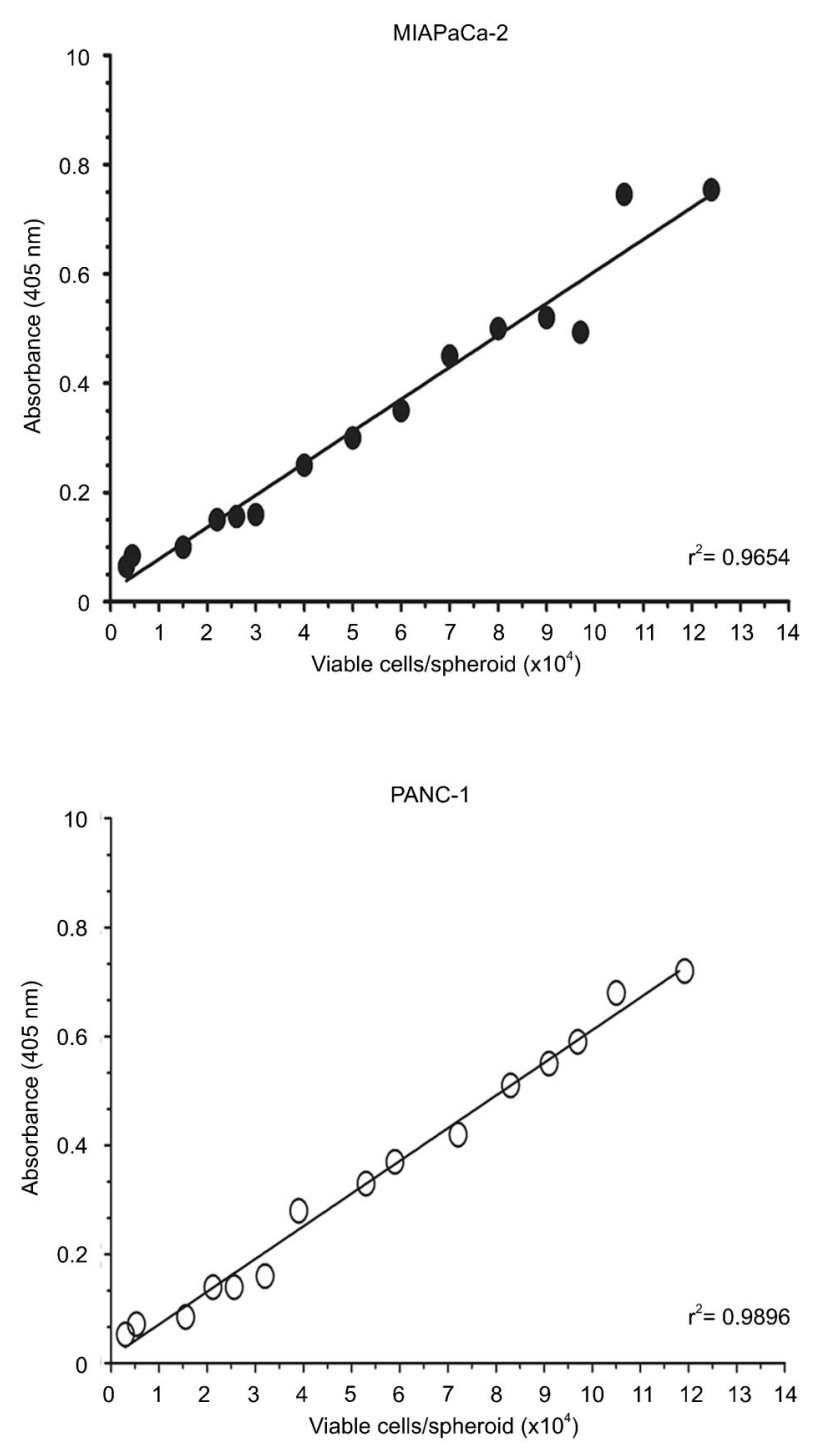

Figure 2. Linearity of the acid phosphatase (APH) assay in MIAPaCa-2 and PANC-1 spheroids. APH colorimetric measurement in MIAPaCa-2 and PANC-1 spheroids (mean, $\mathrm{n} \geqslant 8$ spheroids) as a function of the average number of viable cells per spheroid. Three aliquots of 20 spheroids were measured for each data point.

be supplementary parameters of 3-D cultures when they are used for drug-efficacy testing.

\section{Discussion}

The limitations of 2-D cell culture have motivated researchers to develop an in vitro model for the study of cancer and drug efficacy. Compared to animal models, in vitro models are conducive to systematic, repetitive, and quantitative investigation of cell or tissue physiology in drug discovery and development $(20,21)$. These models can be

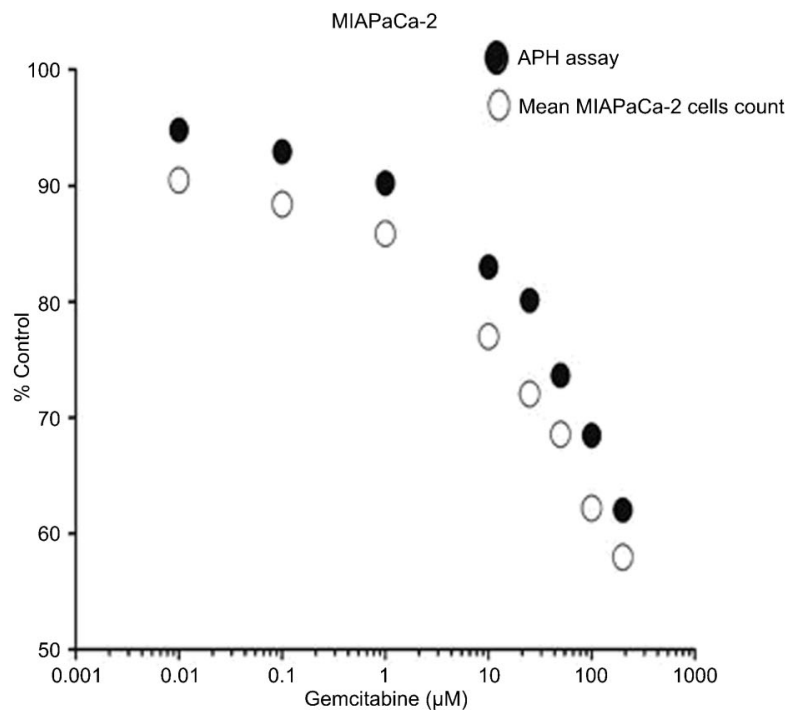

PANC-1

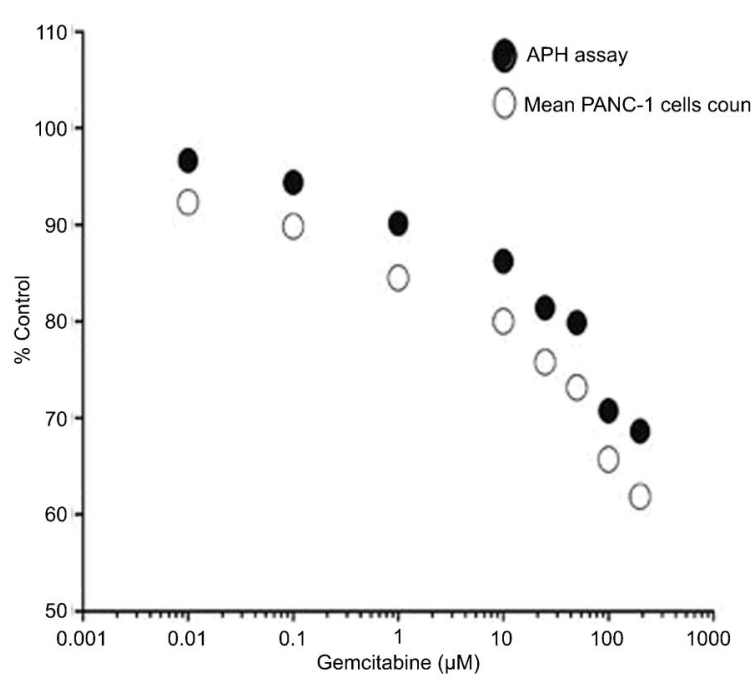

Figure 3. Acid phosphatase (APH) activity reflects cell viability in MIAPaCa-2 and PANC-1 spheroids after treatment. Comparison of APH activity and live cell counts following dissociation in MIAPaCa-2 and PANC-1 spheroids after treatment with different concentrations of gemcitabine for $72 \mathrm{~h}$. Drug efficacy was documented relative to the respective untreated controls. Data are reported as means.

more easily controlled and are usually less expensive and less time-consuming than animal models. In vitro models can be used to assess a large number of combinations of experimental parameters. Such high-throughput testing is usually not feasible with animal-based models. Here, we have established a spheroid-based 3-D culture model in MIAPaCa-2 and PANC-1 MCTS using the APH assay, and investigated the potential of the model as a spheroid-based drug assay for pancreatic cancer in 3-D cultures. 

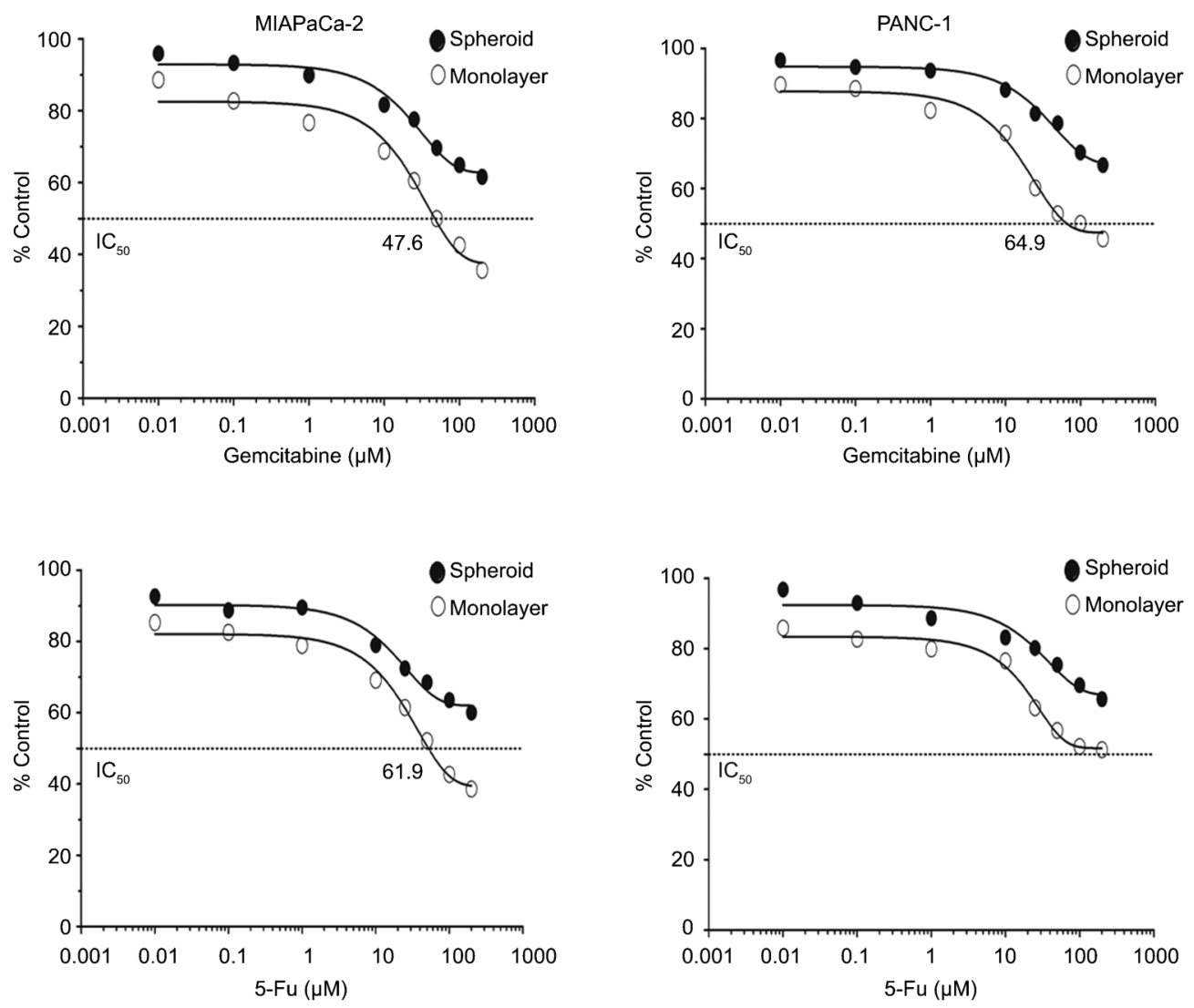

Figure 4. Application of the acid phosphatase (APH) assay to determine drug effects in MIAPaCa-2 and PANC-1 spheroid cultures. APH activity/cell viability in MIAPaCa-2 and PANC-1 monolayer and spheroid cultures after $72 \mathrm{~h}$ of treatment with gemcitabine and 5fluorouracil $(5-\mathrm{FU})$. Data are reported as means of $\geqslant 3$ individual experiments each with eight spheroids treated and measured per condition. $I C_{50}$ values were calculated to investigate the difference of drug efficacy in 2-D vs 3-D culture and in these two pancreatic cancer cell lines.

The MCTS model was first described in the 1970s by Sutherland et al. (5) as a way to mimic the heterogeneity present in solid tumors and account for the effect of the tumor microenvironment on drug transport and efficacy. This model attempted to bridge the gap between standard monolayer cell culture and actual tumors. In fact, tumor cells grown as spheroids acquire some type of clinically relevant multicellular resistance to apoptosis-inducing drugs, which may mimic the chemoresistance found in solid tumors (22-24). Thus, experts in the field have proposed including MCTS as a mandatory model in major programs for drug screening and development. The underlying benefits of using MCTS in antitumor drug testing have frequently been alluded to $(13,25,26)$. In our previous study (27), a 3-D culture method was found for assessing chemosensitivity. However, the spheroids used in that method were not homogeneous, and the complex method was not convenient for antitumor drug testing.

In this study, spheroids grown from MIAPaCa-2 and PANC-1 pancreatic cancer cell lines showed an exponential growth pattern in agarose-treated, 96-well plates.
A maximum spheroid diameter of approximately $1 \mathrm{~mm}$ was reached after about 9 days of culture for MIAPaCa-2 and 10 days for PANC-1 spheroids. The physiological state of spheroids depends on the spheroid size, the individual and cell-type-specific behavior of pancreatic cancer cells, the cell density, and also the culture time. We intended to establish a spheroid-based screen from these two pancreatic cancer cell lines with clear pathophysiological gradients but without obvious cellular death at the initiation of drug treatment. According to the literature and our experience, a 4-day initiation interval for spheroid formation was found to establish spheroids of pancreatic cancer cells reproducibly under identical culture conditions $(15,28)$. The spheroids would reach a standard size of 365 to $410 \mu \mathrm{m}$ after $96 \mathrm{~h}$ of incubation at the initiation of drug treatment. Small spheroids with a size of up to $200 \mu \mathrm{m}$ are often used for drug testing and may be sufficient to reflect 3-D cell-cell and cell-matrix interaction (29-31). It is clearly inappropriate, when attempting to create pathophysiological conditions, to have a hypoxic area in the spheroid center. Hypoxia is not 


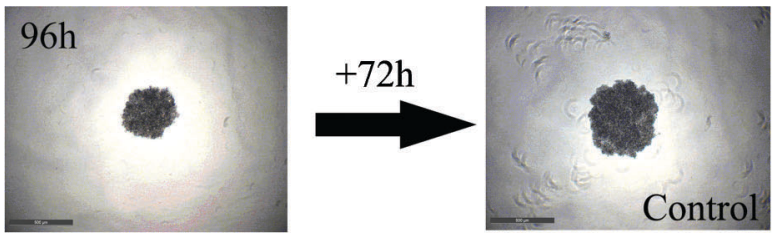

$+72 \mathrm{~h}$ treated with
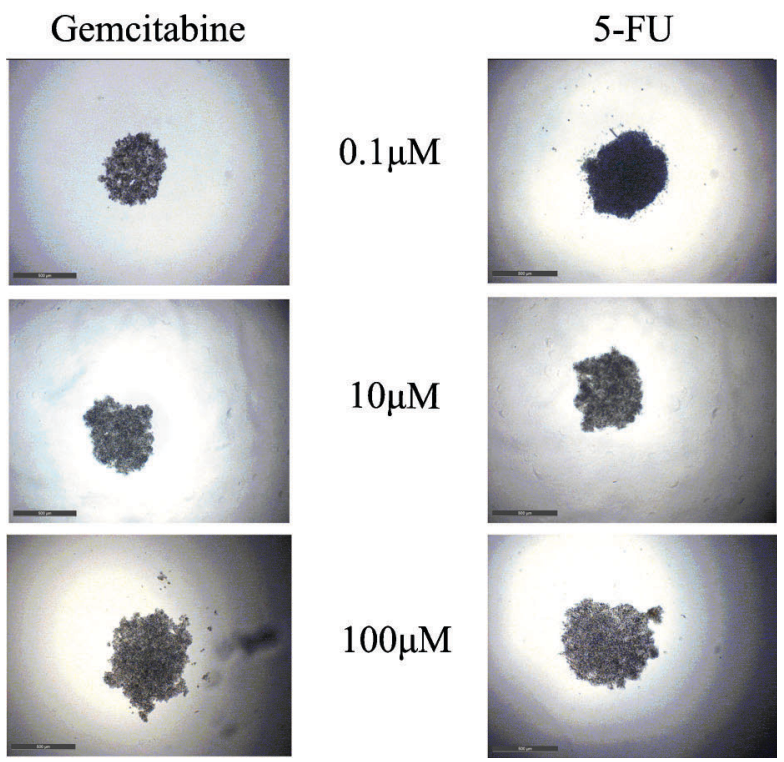

Figure 5. Spheroid integrity following treatment with gemcitabine and 5-fluorouracil (5-FU). Phase contrast images of MIAPaCa-2 spheroids at the initiation of drug treatment and after a 72-h treatment interval with $0.1,10$, and $100 \mu \mathrm{M}$ (Bar: $500 \mu \mathrm{m}$ ).

only a well-established radio- and drug-resistance factor, but also leads to numerous indirect effects in tumor cells by modulating expression patterns $(25,32)$. Larger spheroids with a diameter between 500 and $600 \mu \mathrm{m}$ develop secondary central necroses, which make the pathophysiological conditions complex and difficult to control. Thus, we chose spheroids with a size of $365-410 \mu \mathrm{m}$ for drug treatment.

Easy and rapid handling of both spheroid culture and analysis are crucial requirements of a model for drug testing of pancreatic cancer. Different techniques for spheroid culturing have been extensively described in studies, including advantages and disadvantages $(25,26,33)$. We selected the easy and rapid agaroseoverlay, 96-well plate approach to set up the spheroidbased model.

Several different cytotoxicity/viability assays for 2-D culture, based on absorption, luminescence, or fluorescence, were considered and tested in MCTS. Parameters included cellular uptake of membrane-impermeable dyes such as CCK-8, MTT, Alamar blue, and EtHD-1, release of substrates such as lactate dehydrogenase or glucose-6-phosphate dehydrogenase, activity of mitochondrial dehydrogenases or intracellular esterases, and cellular adenosine triphosphate level and the ratio of ATP/ADP. These commercially available systems have been successfully applied in monolayer cultures in various tumor cell lines, including pancreatic cancer cells (34-37). However, most of these tests could not be easily adapted to application in 3-D culture.

Recently, the APH assay was established as a reliable tool to determine cell viability in complex 3-D culture $(18,33)$. The APH assay is simple, rapid, and highthroughput compatible, as it does not require spheroid dissociation. Our study also verified that the APH assay 1) is applicable for single MIAPaCa-2 and PANC-1 spheroids in 96-well plates, 2) does not require spheroid dissociation, and 3 ) is linear and highly sensitive for MIAPaCa-2 and PANC-1 spheroids up to $1.24 \times 10^{5}$ and $1.19 \times 10^{5}$ cells/spheroid, respectively (both up to a size of $1000 \mu \mathrm{m})$.

To establish a pancreatic cancer-specific, spheroidbased model, we used the APH assay by treating MIAPaCa-2 and PANC-1 spheroids with gemcitabine and 5-FU, two drugs that are commonly used for pancreatic cancer. The efficacy of gemcitabine and 5-FU in monolayer and spheroid cultures was compared. We observed a reduced sensitivity of spheroid cell cultures to gemcitabine and 5-FU compared to monolayer cultures, which should be reflected not only by spheroid integrity and size but also by $\mathrm{APH}$ signals and $\mathrm{APH}$-dependent $\mathrm{IC}_{50}$ values. In the present study, monolayer controls of MIAPaCa-2 and PANC-1 showed dose-response curves and $\mathrm{IC}_{50}$ values consistent with the published literature, both for gemcitabine and 5-FU $(35,38,39)$. Drug effects on MIAPaCa-2 spheroids were shown via the APH assay, but the incubation interval necessary to reach the $\mathrm{IC}_{50}$ demonstrated that spheroids of the MIAPaCa-2 pancreatic cancer cell line were less sensitive to gemcitabine and 5-FU than monolayer culture. Reduced drug efficacy was also seen in PANC-1 in the spheroids compared with monolayer culture. We conclude that our pancreatic cancer spheroid model more closely reflected the efficacy of antitumor drugs. With the APH assay, classic drugs for pancreatic cancer such as gemcitabine and 5-FU could be easily monitored in spheroids and compared with monolayer data. One of the potential applications of the spheroid-APH assay is the testing of single and combined therapeutic strategies with new target-, and/or pathwayspecific treatment modalities.

In conclusion, we established a spheroid-based culture model in the MIAPaCa-2 and PANC-1 pancreatic cancer cell lines for drug testing with the APH assay. The model integrates reproducible, easy, and rapid handling for drug testing of pancreatic cancer. The model would help to fill the gap between monolayer cultures and in vivo models in the process of drug development and testing for pancreatic cancer. 


\section{References}

1. Neoptolemos JP. Adjuvant treatment of pancreatic cancer. Eur J Cancer 2011; 47 (Suppl 3): S378-S380, doi: 10.1016/ S0959-8049(11)70210-6.

2. Griffith LG, Swartz MA. Capturing complex 3D tissue physiology in vitro. Nat Rev Mol Cell Biol 2006; 7: 211224, doi: $10.1038 / \mathrm{nrm} 1858$.

3. Mikos AG, Herring SW, Ochareon P, Elisseeff J, Lu HH, Kandel R, et al. Engineering complex tissues. Tissue Eng 2006; 12: 3307-3339, doi: 10.1089/ten.2006.12.3307.

4. Burdett E, Kasper FK, Mikos AG, Ludwig JA. Engineering tumors: a tissue engineering perspective in cancer biology. Tissue Eng Part B Rev 2010; 16: 351-359, doi: 10.1089/ ten.teb.2009.0676.

5. Sutherland RM, Durand RE. Radiation response of multicell spheroids - an in vitro tumour model. Curr Top Radiat Res $Q$ 1976; 11: 87-139.

6. Durand RE. Multicell spheroids as a model for cell kinetic studies. Cell Tissue Kinet 1990; 23: 141-159.

7. Durand RE, Olive PL. Evaluation of bioreductive drugs in multicell spheroids. Int J Radiat Oncol Biol Phys 1992; 22: 689-692, doi: 10.1016/0360-3016(92)90504-B.

8. Groebe K, Erz S, Mueller-Klieser W. Glucose diffusion coefficients determined from concentration profiles in EMT6 tumor spheroids incubated in radioactively labeled Lglucose. Adv Exp Med Biol 1994; 361: 619-625, doi: 10.1007/978-1-4615-1875-4_114.

9. Sakai S, Inamoto K, Liu Y, Tanaka S, Arii S, Taya M. Multicellular tumor spheroid formation in duplex microcapsules for analysis of chemosensitivity. Cancer Sci 2012; 103: 549-554, doi: 10.1111/j.1349-7006.2011.02187.x.

10. Montel F, Delarue M, Elgeti J, Malaquin L, Basan M, Risler $\mathrm{T}$, et al. Stress clamp experiments on multicellular tumor spheroids. Phys Rev Lett 2011; 107: 188102, doi: 10.1103/ PhysRevLett.107.188102.

11. Ho WJ, Pham EA, Kim JW, Ng CW, Kim JH, Kamei DT, et al. Incorporation of multicellular spheroids into 3-D polymeric scaffolds provides an improved tumor model for screening anticancer drugs. Cancer Sci 2010; 101: 2637-2643, doi: 10.1111/j.1349-7006.2010.01723.x.

12. Kruttwig K, Brueggemann C, Kaijzel E, Vorhagen S, Hilger $\mathrm{T}$, Lowik $\mathrm{C}$, et al. Development of a three-dimensional in vitro model for longitudinal observation of cell behavior: monitoring by magnetic resonance imaging and optical imaging. Mol Imaging Biol 2010; 12: 367-376, doi: 10.1007/ s11307-009-0289-x.

13. Kunz-Schughart LA, Freyer JP, Hofstaedter F, Ebner R. The use of 3-D cultures for high-throughput screening: the multicellular spheroid model. J Biomol Screen 2004; 9: 273285, doi: 10.1177/1087057104265040.

14. Friedrich J, Ebner R, Kunz-Schughart LA. Experimental anti-tumor therapy in 3-D: spheroids - old hat or new challenge? Int J Radiat Biol 2007; 83: 849-871, doi: 10.1080/09553000701727531.

15. McLeod EJ, Beischer AD, Hill JS, Kaye AH. Multicellular tumor spheroids grown from pancreatic carcinoma cell lines: use as an orthotopic xenograft in athymic nude mice. Pancreas 1997; 14: 237-248, doi: 10.1097/00006676199704000-00004.

16. Sweigert SE, Alpen EL. Dynamics of cell kinetic parameters during 9L spheroid growth. Cell Tissue Kinet 1986; 19: 567576.

17. Sutherland RM, McCredie JA, Inch WR. Growth of multicell spheroids in tissue culture as a model of nodular carcinomas. J Natl Cancer Inst 1971; 46: 113-120.

18. Friedrich J, Eder W, Castaneda J, Doss M, Huber E, Ebner $R$, et al. A reliable tool to determine cell viability in complex 3-D culture: the acid phosphatase assay. J Biomol Screen 2007; 12: 925-937, doi: 10.1177/1087057107306839.

19. Kunz-Schughart LA, Groebe K, Mueller-Klieser W. Threedimensional cell culture induces novel proliferative and metabolic alterations associated with oncogenic transformation. Int J Cancer 1996; 66: 578-586, doi: 10.1002/(SICI)10970215(19960516)66:4<578::AID-IJC25>3.0.CO;2-2.

20. Drewitz $M$, Helbling $M$, Fried $N$, Bieri $M$, Moritz $W$, Lichtenberg $\mathrm{J}$, et al. Towards automated production and drug sensitivity testing using scaffold-free spherical tumor microtissues. Biotechnol J 2011; 6: 1488-1496, doi: 10.1002/biot.201100290.

21. Cichon MA, Gainullin VG, Zhang Y, Radisky DC. Growth of lung cancer cells in three-dimensional microenvironments reveals key features of tumor malignancy. Integr Biol 2012; 4: 440-448, doi: 10.1039/c1ib00090j.

22. Barbone D, Yang TM, Morgan JR, Gaudino G, Broaddus VC. Mammalian target of rapamycin contributes to the acquired apoptotic resistance of human mesothelioma multicellular spheroids. J Biol Chem 2008; 283: 1302113030, doi: 10.1074/jbc.M709698200.

23. Al-Haij M, Becker MW, Wicha M, Weissman I, Clarke MF Therapeutic implications of cancer stem cells. Curr Opin Genet Dev 2004; 14: 43-47, doi: 10.1016/j.gde.2003.11.007.

24. Desoize B, Jardillier J. Multicellular resistance: a paradigm for clinical resistance? Crit Rev Oncol Hematol 2000; 36: 193-207, doi: 10.1016/S1040-8428(00)00086-X.

25. Hirschhaeuser F, Menne H, Dittfeld C, West J, MuellerKlieser W, Kunz-Schughart LA. Multicellular tumor spheroids: an underestimated tool is catching up again. $J$ Biotechnol 2010; 148: 3-15, doi: 10.1016/j.jbiotec.2010.01.012.

26. Elliott NT, Yuan F. A review of three-dimensional in vitro tissue models for drug discovery and transport studies. $J$ Pharm Sci 2011; 100: 59-74, doi: 10.1002/jps.22257.

27. Liao Q, Hu Y, Zhao YP, Zhou T, Zhang Q. Assessment of pancreatic carcinoma cell chemosensitivity using a threedimensional culture system. Chin Med J 2010; 123: 1871-1877.

28. Sempere LF, Gunn JR, Korc M. A novel 3-dimensional culture system uncovers growth stimulatory actions by TGF $\beta$ in pancreatic cancer cells. Cancer Biol Ther 2011; 12: 198-207, doi: 10.4161/cbt.12.3.15979.

29. Enmon R, Yang WH, Ballangrud AM, Solit DB, Heller G, Rosen N, et al. Combination treatment with 17-N-allylamino17-demethoxy geldanamycin and acute irradiation produces supra-additive growth suppression in human prostate carcinoma spheroids. Cancer Res 2003; 63: 8393-8399.

30. Fehlauer F, Muench M, Rades D, Stalpers LJ, Leenstra S, van der Valk $\mathrm{P}$, et al. Effects of irradiation and cisplatin on human glioma spheroids: inhibition of cell proliferation and cell migration. J Cancer Res Clin Oncol 2005; 131: 723-732, doi: 10.1007/s00432-005-0014-3.

31. Lambert B, de Ridder L, Slegers G, De Gelder V, Dierckx 
RA, Thierens H. Screening for supra-additive effects of cytotoxic drugs and gamma irradiation in an in vitro model for hepatocellular carcinoma. Can J Physiol Pharmacol 2004; 82: 146-152, doi: 10.1139/y04-008.

32. Shield K, Ackland ML, Ahmed N, Rice GE. Multicellular spheroids in ovarian cancer metastases: Biology and pathology. Gynecol Oncol 2009; 113: 143-148, doi: 10.1016/j.ygyno.2008.11.032.

33. Friedrich J, Seidel C, Ebner R, Kunz-Schughart LA Spheroid-based drug screen: considerations and practical approach. Nat Protoc 2009; 4: 309-324, doi: 10.1038/ nprot.2008.226.

34. Khaitan D, Chandna S, Arya MB, Dwarakanath BS. Establishment and characterization of multicellular spheroids from a human glioma cell line; Implications for tumor therapy. J Transl Med 2006; 4: 12, doi: 10.1186/1479-5876-4-12.

35. Arumugam $\mathrm{T}$, Ramachandran $\mathrm{V}$, Fournier KF, Wang $\mathrm{H}$, Marquis L, Abbruzzese JL, et al. Epithelial to mesenchymal transition contributes to drug resistance in pancreatic cancer. Cancer Res 2009; 69: 5820-5828, doi: 10.1158/
0008-5472.CAN-08-2819.

36. Shah AN, Summy JM, Zhang J, Park SI, Parikh NU, Gallick GE. Development and characterization of gemcitabineresistant pancreatic tumor cells. Ann Surg Oncol 2007; 14: 3629-3637, doi: 10.1245/s10434-007-9583-5.

37. Akada M, Crnogorac-Jurcevic T, Lattimore S, Mahon P, Lopes R, Sunamura M, et al. Intrinsic chemoresistance to gemcitabine is associated with decreased expression of BNIP3 in pancreatic cancer. Clin Cancer Res 2005; 11: 3094-3101, doi: 10.1158/1078-0432.CCR-04-1785.

38. Zhang $Q$, Zhao YP, Liao Q, Hu Y, Xu Q, Zhou L, et al. Associations between gene polymorphisms of thymidylate synthase with its protein expression and chemosensitivity to 5 -fluorouracil in pancreatic carcinoma cells. Chin Med $\mathrm{J}$ 2011; 124: 262-267.

39. Kurata N, Fujita H, Ohuchida K, Mizumoto K, Mahawithitwong $\mathrm{P}$, Sakai $\mathrm{H}$, et al. Predicting the chemosensitivity of pancreatic cancer cells by quantifying the expression levels of genes associated with the metabolism of gemcitabine and 5fluorouracil. Int J Oncol 2011; 39: 473-482. 